\title{
ON SOME NEW CONGRUENCES IN THE THEORY OF BERNOULLI'S NUMBERS
}

N. G. W. H. BEEGER

For Bernoulli's numbers the following relations are known:

$$
\begin{array}{ll}
(h+1)^{n}=h^{n} ; \quad & n>1, \quad h_{1}=-\frac{1}{2}, \quad B_{n}=(-1)^{n-1} h_{2 n} \\
& h_{2 n+1}=0 \quad \text { for } n>0 .
\end{array}
$$

For the symbol $k^{n}=h^{n+1} /(n+1)$ Kummer proved the congruence

$$
k^{a}\left(1-k^{b}\right)^{c} \equiv 0\left(\bmod \left(p^{a}, p^{e c}\right)\right),
$$

$p$ being a prime, $b=p^{e-1}(p-1) b_{1}, a+1 \not \equiv 0(\bmod (p-1))$. G. Frobenius* has given another proof of this congruence, without using infinite series. I shall now prove the congruence

$$
\begin{array}{r}
(-1)^{i-1} k^{a+m b} \equiv \sum_{s=1}^{i}(-1)^{s-1} C_{m, s-1} C_{m-s, i-s} k^{a+(s-1) b}\left(\bmod p^{i}\right) \\
b=p-1,
\end{array}
$$

which is equivalent to

$$
(-1)^{i-1} \frac{B_{n+m \mu}}{2 n+2 m \mu} \equiv \sum_{s=1}^{i}(-1)^{s-1+(m-s+1) \mu}
$$

$$
\text { - } C_{m, s-1} C_{m-s, i-s} \frac{B_{n+(s-1) \mu}}{2 n+2(s-1) \mu}\left(\bmod p^{i}\right),
$$

$$
C_{m, 0}=1, m \geqq i, i<2 n-1,2 n \not \equiv 0(\bmod (p-1)), \mu=(p-1) / 2 .
$$

Take, in (1), $b=p-1, c=i, a=2 n-1$; then (1) gives

$$
(-1)^{i-1} k^{a+b i} \equiv \sum_{s=1}^{i}(-1)^{s-1} C_{i, s-1} k^{a+(s-1) b}\left(\bmod p^{i}\right) .
$$

Hence (2) is proved for the case $m=i$. Now suppose that (2) is proved for $m=i, i+1, i+2, \cdots, m$. From (1) it follows that

$$
\begin{aligned}
(-1)^{m} k^{a+(m+1) b} \equiv & \sum_{s=1}^{i}(-1)^{s-1} C_{m+1, s-1} k^{a+(s-1) b} \\
& +\sum_{s=i+1}^{m+1}(-1)^{s-1} C_{m+1, s-1} k^{a+(s-1) b}\left(\bmod p^{m+1}\right) .
\end{aligned}
$$

* Sitzungsberichte der Preussischen Akademie, vol. 39 (1910), p. 809 
By substituting, for each term of the second sum in the right-hand side of (4), the series from (2), we obtain

$$
\begin{aligned}
(-1)^{m} k^{a+(m+1) b} \equiv & \sum_{s=1}^{i}(-1)^{s-1} k^{a+(s-1) b}\left(C_{m+1, s-1}\right. \\
& -C_{m+1, i} C_{i, s-1} C_{i-s, i-s}+C_{m+1, i+1} C_{i+1, s-1} C_{i-s+1, i-s} \\
& \left.-\cdots \pm C_{m+1, m} C_{m, s-1} C_{m-s, i-s}\right)\left(\bmod p^{i}\right) .
\end{aligned}
$$

Let the coefficient of $k^{a}$ be denoted by $S_{m}$. Then

$$
\begin{aligned}
S_{m}=1 & -C_{m+1, i} C_{i-1, i-1}+C_{m+1, i+1} C_{i, i-1} \\
& -\cdots+(-1)^{m+i-1} C_{m+1, m} C_{m-1, i-1},
\end{aligned}
$$

and, using the known relation

we have

$$
C_{m+1, c}-C_{m, c}=C_{m, c-1},
$$

$$
\begin{aligned}
S_{m}-S_{m-1}= & \sum_{j=i}^{m-1}(-1)^{j-i+1} C_{m, j-1} C_{j-1, i-1}+(-1)^{m+i-1} C_{m+1, m} C_{m-1, i-1} \\
= & C_{m, i-1} \sum_{j=i}^{m-1}(-1)^{i-i+1} C_{m-i+1, m-j+1} \\
& +(-1)^{m+i-1} C_{m+1, m} C_{m-1, i-1} \\
= & (-1)^{m-i+1}\left(C_{m, i-1}+C_{m-1, i-1}\right) .
\end{aligned}
$$

From (6) it follows that $S_{i}=-i$; hence from (7) we have

$$
\begin{aligned}
S_{m}-S_{i}= & C_{i, i-1}-C_{i+1, i-1}+\cdots+(-1)^{m-i+1} C_{m-1, i-1} \\
& +\left(C_{i+1, i-1}-C_{i+2, i-1}+\cdots+(-1)^{m-i+1} C_{m, i-1}\right), \\
S_{m}= & (-1)^{m-i+1} C_{m, i-1} .
\end{aligned}
$$

Let further the coefficient of $k^{a+j b}$ in the second member of (5) be denoted by $S_{m}{ }^{\prime}$; then

$$
\begin{aligned}
S_{m}^{\prime}= & C_{m+1, j}-C_{m+1, j} C_{i, j} C_{i-j-1, i-j-1}+\cdots \pm C_{m+1, m} C_{m, j} C_{m-j-1, i-j-1} \\
= & C_{m+1, j}\left(1-C_{m-j+1, i-j} C_{i-j-1, i-j-1}+C_{m-j+1, i-j+1} C_{i-j, i-j-1}\right. \\
& \left.-\cdots \pm C_{m-j+1, m-j} C_{m-j-1, i-j-1}\right)
\end{aligned}
$$

hence

$$
S_{m}^{\prime}=C_{m+1, j} S_{m-j}=C_{m+1, j} C_{m-j, i-j-1}(-1)^{m-i+1}
$$

by (8). From (5) the congruence (2) is now proved for $(m+1)$; hence (2) is true in general for all numbers $m=i, i+1, \cdots$. 
In order to get a congruence analogous to (2) and (3), but for a modulus which is a higher power of $p$ than $p^{a}$, take, in (2), $a+j b$ in place of $a$, and replace $(m+j)$ by $m$. We have

$$
\begin{aligned}
(-1)^{i-1} k^{a+m b} & \equiv \sum_{s=1}^{i}(-1)^{s-1} C_{m-j, s-1} C_{m-j-s, i-s} k^{a+(s+j-1) b}\left(\bmod p^{i}\right), \\
& m \geqq i+j, i \leqq a+j b, a+1 \not \equiv 0(\bmod (p-1)),
\end{aligned}
$$

with the equivalent relation

$$
(-1)^{i-1} \frac{B_{n+m \mu}}{2 n+2 m \mu} \equiv \sum_{s=1}^{i}(-1)^{s-1+(m-s+1) \mu}
$$

$$
\begin{array}{r}
\cdot C_{m-j, s-1} C_{m-j-s, i-s} \frac{B_{n+(s+j-1) \mu}}{2 n+2 \mu(s+j-1)}\left(\bmod p^{i}\right), \\
m \geqq i+j, i \leqq 2 n-1+j(p-1), 2 n \neq 0(\bmod (p-1)) .
\end{array}
$$

A prime $p>3$ is said to be irregular if it divides one of the numbers $B_{1}, B_{2}, \cdots, B_{\mu-1}$, say $B_{n}$. It is known by (1) that in this case each number $B_{n+m \mu}$ is divisible by $p$.

THEOREM. If $p$ is an irregular prime, and if $k^{a} \equiv 0(\bmod p)$, then for each number $i$ the positive integers $m_{1}, m_{2}, \cdots, m_{i-1}<p$, can be determined uniquely by the chain of congruences

$$
\begin{aligned}
& k^{a} \equiv m_{1} P\left(\bmod p^{2}\right), \\
& k^{a+m_{1} b} \equiv m_{2} p P\left(\bmod p^{3}\right), \\
& k^{a+\left(m_{1}+m_{2} p\right) b} \equiv m_{3} p^{2} P\left(\bmod p^{4}\right), \\
& k^{a+\left(m_{1}+m_{2} p+\cdots+m_{i-2} p^{i-3}\right) b} \equiv m_{i-1} p^{i-2} P\left(\bmod p^{i}\right),
\end{aligned}
$$

provided that

$$
P=k^{a}-k^{a+b} \not \equiv 0\left(\bmod p^{2}\right)
$$

consequently

$$
k^{a+\left(m_{1}+m_{2} p+\cdots+m_{i-1} p^{i-2}\right) b} \equiv 0\left(\bmod p^{i}\right) .
$$

In the above $k$ is defined as at the beginning of the article, and $b=p-1$.

PROOF. In the congruence (2) take $i=2$; this gives

$$
\begin{aligned}
-k^{a+m b} & \equiv(m-1) k^{a}-m k^{a+b}\left(\bmod p^{2}\right), \\
k^{a+m b} & \equiv k^{a}-m\left(k^{a}-k^{a+b}\right)\left(\bmod b^{2}\right) .
\end{aligned}
$$


The congruence

$$
k^{a}-m\left(k^{a}-k^{a+b}\right) \equiv 0\left(\bmod p^{2}\right),
$$

wherein $k^{a}$ and $k^{a+b}$ are divisible by $p$, has one solution $m_{1}<p$ if and only if $P=k^{a}-k^{a+b} \not \equiv 0\left(\bmod p^{2}\right)$, and it follows from (11) that

$$
k^{a+m_{1} b} \equiv 0\left(\bmod p^{2}\right) .
$$

Hence the theorem is proved for $i=2$. Suppose that it is proved for $2,3,4, \cdots, i$, and put

$$
m_{1}+m_{2} p+\cdots+m_{i-2} p^{i-3}=m^{\prime}
$$

then $k^{a+m^{\prime} b} \equiv 0\left(\bmod p^{i}\right)$. Now take the congruence $(9)$ for $(i+1)$ in place of $i$, which gives

$(-1)^{i} k^{a+m b} \equiv \sum_{s=1}^{i+1}(-1)^{s-1} C_{m-j, s-1} C_{m-j-s, i+1-s} k^{a+(s+j-1) b}\left(\bmod p^{i+1}\right)$.

Let the polynomial in the right-hand side be denoted by $G(m)$. Then

$$
G\left(m^{\prime}+p^{i-1} x\right) \equiv G\left(m^{\prime}\right)+p^{i-1} x G^{\prime}\left(m^{\prime}\right)\left(\bmod p^{i+1}\right) ;
$$

also

$$
G\left(m^{\prime}\right) \equiv(-1)^{i} k^{a+m^{\prime} b}\left(\bmod p^{i+1}\right)
$$

from the definition of $G$, and (2) gives, for $i=2$,

$$
G(m)=(-1)^{i} k^{a+m b} \equiv(-1)^{i-1}\left\{(m-1) k^{a}-m k^{a+b}\right\}\left(\bmod p^{2}\right) ;
$$

hence

$$
G^{\prime}(m) \equiv(-1)^{i-1}\left\{k^{a}-k^{a+b}\right\}\left(\bmod p^{2}\right),
$$

and setting $m=m^{\prime}$ in this relation, we get from (11a)

$$
G\left(m^{\prime}+p^{i-1} x\right) \equiv(-1)^{i} k^{a+m^{\prime} b}+(-1)^{i-1} p^{i-1} x\left(k^{a}-k^{a+b}\right)\left(\bmod p^{i+1}\right),
$$

and hence

$(-1)^{i} k^{a+\left(m^{\prime}+p^{i-1} x\right) b} \equiv(-1)^{i} k^{a+m^{\prime} b}+(-1)^{i-1} p^{i-1} x\left(k^{a}-k^{a+b}\right)\left(\bmod p^{i+1}\right)$.

Now $k^{a+m^{\prime} b} \equiv 0\left(\bmod p^{i}\right)$. The congruence

$$
k^{a+m^{\prime} b}-p^{i-1} x\left(k^{a}-k^{a+b}\right) \equiv 0\left(\bmod p^{i+1}\right)
$$

has therefore one solution $x=m_{i}<p$ if and only if $k^{a}-k^{a+b} \not \equiv 0$ $\left(\bmod p^{2}\right)$, and then $k^{a+\left(m^{\prime}+p^{i-1} m_{i}\right) b} \equiv 0\left(\bmod p^{i+1}\right)$. The theorem is proved for $(i+1)$ and hence is true for all values of $i$.

It follows immediately from this theorem that for each number $i$, as large as we please, the numbers $m_{1}, m_{2}, \cdots, m_{i-1}$ can be deter- 
mined so that if $B_{n} \equiv 0(\bmod p), n<p, p$ an irregular prime, then

$$
B_{n+\left(m_{1}+m_{2} p+\cdots+m_{i-1} p^{i-2}\right) \mu} \equiv 0\left(\bmod p^{i}\right) \text {, }
$$

if

$$
\frac{B_{n}}{2 n} \not \equiv(-1)^{(p-1) / 2} \frac{B_{n+(p-1) / 2}}{2 n+p-1} .
$$

Pollaczek* calculated, in the cases $n=16, p=37 ; n=22, p=59$; and $n=29, p=67$, the number $m_{1}$ for which $B_{n+m_{1} \mu} \equiv 0\left(\bmod p^{2}\right)$. His calculations gave me the idea to construct my congruences (3) and (10) and to formulate the theorem.

The substitution of $m=2 n$ in (3) gives the result

$$
\begin{aligned}
(-1)^{i-1} \frac{B_{n p}}{n p} & \equiv \sum_{s=1}^{i}(-1)^{(s-1)(\mu+1)} C_{2 n, s-1} C_{2 n-s, i-s} \\
& \cdot \frac{B_{n+(s-1) \mu}}{2 n+2(s-1) \mu}\left(\bmod p^{i}\right), 2 n \neq 0(\bmod (p-1)) .
\end{aligned}
$$

The case $i=2$ is of special interest. H. S. Vandiver and his collaborators, in their researches about the second case of Fermat's last theorem, $\dagger$ have made very extensive calculations to find the residues of $B_{n p}$, modulo $p^{3}, p$ being an irregular prime less than 211 , not knowing the congruence $(12)$. For $B_{n} \equiv 0(\bmod p)$, we have $n<\mu$; then $B_{n p} \equiv 0$ $\left(\bmod p^{2}\right)$, and $(12)$ gives, for $i=2$,

$$
B_{n p} \equiv-\frac{p}{2 n-1}\left\{(2 n-1)^{2} B_{n}-(-1)^{(p-1) / 2}(2 n)^{2} B_{n+\mu}\right\}\left(\bmod p^{3}\right) .
$$

Using the existing tables of Bernoulli's numbers we can obtain from this congruence the residue of $B_{n p}$, modulo $p^{3}$, after a simple calculation. Thus I have checked the results of Vandiver (except for $p=157$ and $149 ; B_{133}$ and $B_{139}$ not being in the tables) and have found them all correct.

Amsterdam, Holland

* Mathematische Zeitschrift, vol. 21 (1924), pp. 28-31. Some of his results are wrong. They should be $B_{22} \equiv 50 \cdot 59, B_{51} \equiv 42 \cdot 59\left(\bmod 59^{2}\right), B_{62} \equiv 37 \cdot 67\left(\bmod 67^{2}\right)$.

$\dagger$ Transactions of this Society, vol. 31 (1929), pp. 613, 639-642. 